\title{
Development of an inside-out augmented reality technique for neurosurgical navigation
}

\author{
*Yun-Sik Dho, MD, ${ }^{1}$ Sang Joon Park, PhD, ${ }^{2}$ Haneul Choi, BA, ${ }^{2}$ Youngdeok Kim, BS, ${ }^{2}$ \\ Hyeong Cheol Moon, PhD, ${ }^{1}$ Kyung Min Kim, MD, ${ }^{3}$ Ho Kang, MD, ${ }^{3}$ Eun Jung Lee, MD, ${ }^{3}$ \\ Min-Sung Kim, MD, ${ }^{3}$ Jin Wook Kim, MD, PhD, ${ }^{3}$ Yong Hwy Kim, MD, PhD, ${ }^{3}$ \\ Young Gyu Kim, MD, PhD, ${ }^{1}$ and Chul-Kee Park, MD, PhD ${ }^{3}$
}

1Department of Neurosurgery, Chungbuk National University Hospital, Chungbuk National University College of Medicine, Cheongju, Republic of Korea; ${ }^{2 M E D I C A L I P ~ C o . ~ L t d ., ~ S e o u l, ~ R e p u b l i c ~ o f ~ K o r e a ; ~ a n d ~}{ }^{3}$ Department of Neurosurgery, Seoul National University Hospital, Seoul National University College of Medicine, Seoul, Republic of Korea

\begin{abstract}
OBJECTIVE With the advancement of 3D modeling techniques and visualization devices, augmented reality (AR)based navigation (AR navigation) is being developed actively. The authors developed a pilot model of their newly developed inside-out tracking AR navigation system.

METHODS The inside-out AR navigation technique was developed based on the visual inertial odometry (VIO) algorithm. The Quick Response (QR) marker was created and used for the image feature-detection algorithm. Inside-out AR navigation works through the steps of visualization device recognition, marker recognition, AR implementation, and registration within the running environment. A virtual 3D patient model for AR rendering and a 3D-printed patient model for validating registration accuracy were created. Inside-out tracking was used for the registration. The registration accuracy was validated by using intuitive, visualization, and quantitative methods for identifying coordinates by matching errors. Fine-tuning and opacity-adjustment functions were developed.

RESULTS ARKit-based inside-out AR navigation was developed. The fiducial marker of the AR model and those of the 3D-printed patient model were correctly overlapped at all locations without errors. The tumor and anatomical structures of AR navigation and the tumors and structures placed in the intracranial space of the 3D-printed patient model precisely overlapped. The registration accuracy was quantified using coordinates, and the average moving errors of the $x$-axis and $y$-axis were $0.52 \pm 0.35$ and $0.05 \pm 0.16 \mathrm{~mm}$, respectively. The gradients from the $x$-axis and $y$-axis were $0.35^{\circ}$ and $1.02^{\circ}$, respectively. Application of the fine-tuning and opacity-adjustment functions was proven by the videos.

CONCLUSIONS The authors developed a novel inside-out tracking-based AR navigation system and validated its registration accuracy. This technical system could be applied in the novel navigation system for patient-specific neurosurgery.

https://thejns.org/doi/abs/10.3171/2021.5.FOCUS21184
\end{abstract}

KEYWORDS augmented reality; navigation; brain tumor; surgical planning; inside-out tracking

$\mathrm{N}$ EURONAVIGATION systems were introduced in 1986 and are currently essential tools in the field of neurosurgery. ${ }^{1}$ By specifying the location of intracranial lesions through matching 2D MR images with 3D patient anatomy, neuronavigation enables precise and safe neurosurgery. ${ }^{2-4}$ Neuronavigation systems have remarkably improved in their accuracy and multitasking capacity since the first models, although dependence on 2D screen images for identifying lesion locations has persisted. To use neuronavigation to match lesions on MR images with the correct locations in the brain, fiducial markers are routinely attached to the patient to preoperatively perform MR image registration and should remain attached to the patient's head until entry into the operating theater..$^{5-7}$ The fiducial marker tracking method of the contemporary system is based on the outside-in tracking method, in which

ABBREVIATIONS AR = augmented reality; HMD = head-mounted display; IMU = inertial measurement unit; $\mathrm{QR}=\mathrm{Quick}$ Response; VI SLAM = visual inertial simultaneous localization and mapping; VIO = visual inertial odometry.

SUBMITTED March 26, 2021. ACCEPTED May 25, 2021.

INCLUDE WHEN CITING DOI: 10.3171/2021.5.FOCUS21184.

* Y.S.D. and S.J.P. contributed equally to this work. 

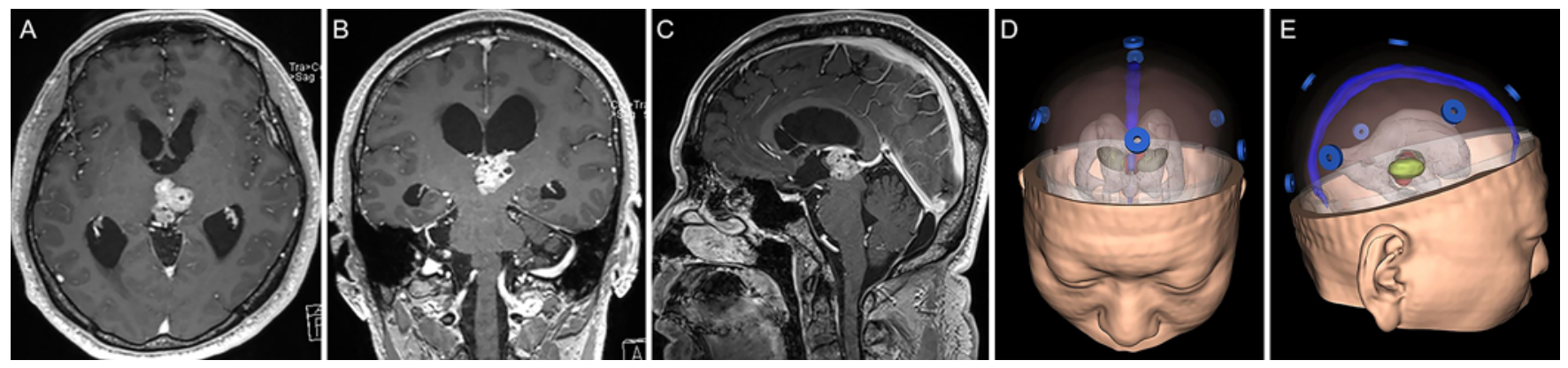

FIG. 1. The preoperative MR images obtained from the patient and the virtual 3D model for AR navigation. A: Axial plane. B: Coronal plane. C: Sagittal plane. D: Anterior view. E: Lateral view. In panels D and E, the tumor (red) is located in the third ventricle (gray) between the thalami (green). Registration markers and superior sagittal sinus are shown in blue. The opacities of skin and bone were adjusted for the visualization of intracranial structures.

the camera and sensor are held in a specific position and then markers and matching targets are tracked through the camera and sensor. ${ }^{8-10}$ This method requires the camera and sensor to be placed in a high position to track spherical markers covered with retroreflective materials, whereas the cranial reference array for mounting markers should be fixed near the patient to maintain registration.

Recently developed image segmentation and rendering technologies have facilitated the production of 3D brain virtual models and the subsequent introduction of several augmented reality (AR) navigation systems using these 3D virtual models. ${ }^{11-13}$ AR navigation systems use various visualization devices, such as video projectors, tablet PCs, and head-mounted displays (HMDs). However, most of the systems incorporate the classic outside-in tracking methods. ${ }^{14-16}$ Outside-in tracking AR navigations have the advantage that the view of the surgical field need not be alternated with the screen of the navigation system, which uses 3D images superimposed on a matching target. However, similar to conventional neuronavigation, these newer systems require a time-consuming cumbersome registration process without allowing positional change of markers. ${ }^{14-16}$ Therefore, outside-in tracking-based AR navigations may develop misalignment after the mobilization of the visualization device and the markers after initial registration.

The inside-out tracking method implies a continuous tracing of tracking markers, matching targets, and surrounding environments from the user's location through cameras and sensors mounted simultaneously on the device used for visualization. ${ }^{17}$ With the advanced development of visualization devices, such as tablet PC and HMD, there are active attempts to apply these devices to AR navigation. ${ }^{18,19}$ Compared to outside-in tracking, inside-out tracking requires only the equipment for visualization and a feature-detection marker for registration, with the associated advantage of requiring relatively simple equipment and a resultant low system configuration cost. However, inside-out tracking-based AR navigation was previously reported to exhibit less accuracy than outside-in tracking navigation, and consequently integration of the inside-out tracking technique into neurosurgical practice was presumed to be premature. ${ }^{18,19}$ If technical improvement of the inside-out tracking method for AR navigation could ensure improved registration accuracy and adjustment of the registration error, then simpler and lighter AR navigation systems could be developed. We developed ARKit (Apple Inc.)-based software that enables inside-out tracking and mounted it to an iPad (Apple Inc.) to test the feasibility of developing a clinically usable inside-out tracking AR neuronavigation system.

\section{Methods}

\section{D Model Segmentation}

The 3D patient model for AR navigation was created using segmentation, reconstruction, and a rendering program that the authors had previously developed and released (MEDIP, http://medicalip.com/shop/medip.php, MEDICALIP). The above-mentioned processes were performed with the approval of the institutional review board of the institution where the case originated. Enhanced T1weighted, T2-weighted, T2 fluid-attenuated inversion recovery (FLAIR) MR and CT images (1.0-mm-thick slabs and $1.0-\mathrm{mm}$ spaces) were used in combination as source images for segmentation. The preoperative MR image of the patient is shown in Fig. 1, and the segmentation process in the program of the 3D patient model is described in Supplemental Material. The virtual 3D model for AR navigation is implemented with a $3 \mathrm{D}$ visualization of the brain tumor and the anatomical structures surrounding the brain tumor, brain parenchyma, skull (including the upper face and cranial regions of the supra-maxillary area), skin, and fiducial markers attached to the skin (Fig. 1D and E; http://medicalip.synology.me:8080/STLRendering/ 210122_AR_case01_v1.html). Moreover, the virtual 3D model was used to produce a 3D-printed brain tumor and patient model the same size as the actual size of the patient who was used as a registration target to validate the registration accuracy of the AR navigation and simulate the real-life demonstration.

\section{Algorithm and Workflow of Inside-Out AR Navigation}

Inside-out AR navigation was developed using ARKit as a framework for AR. The workflow of the inside-out AR navigation algorithm (Fig. 2) comprises 3 stages: 1) visualization device recognition, 2) marker recognition, and 3) AR implementation and registration within the running 


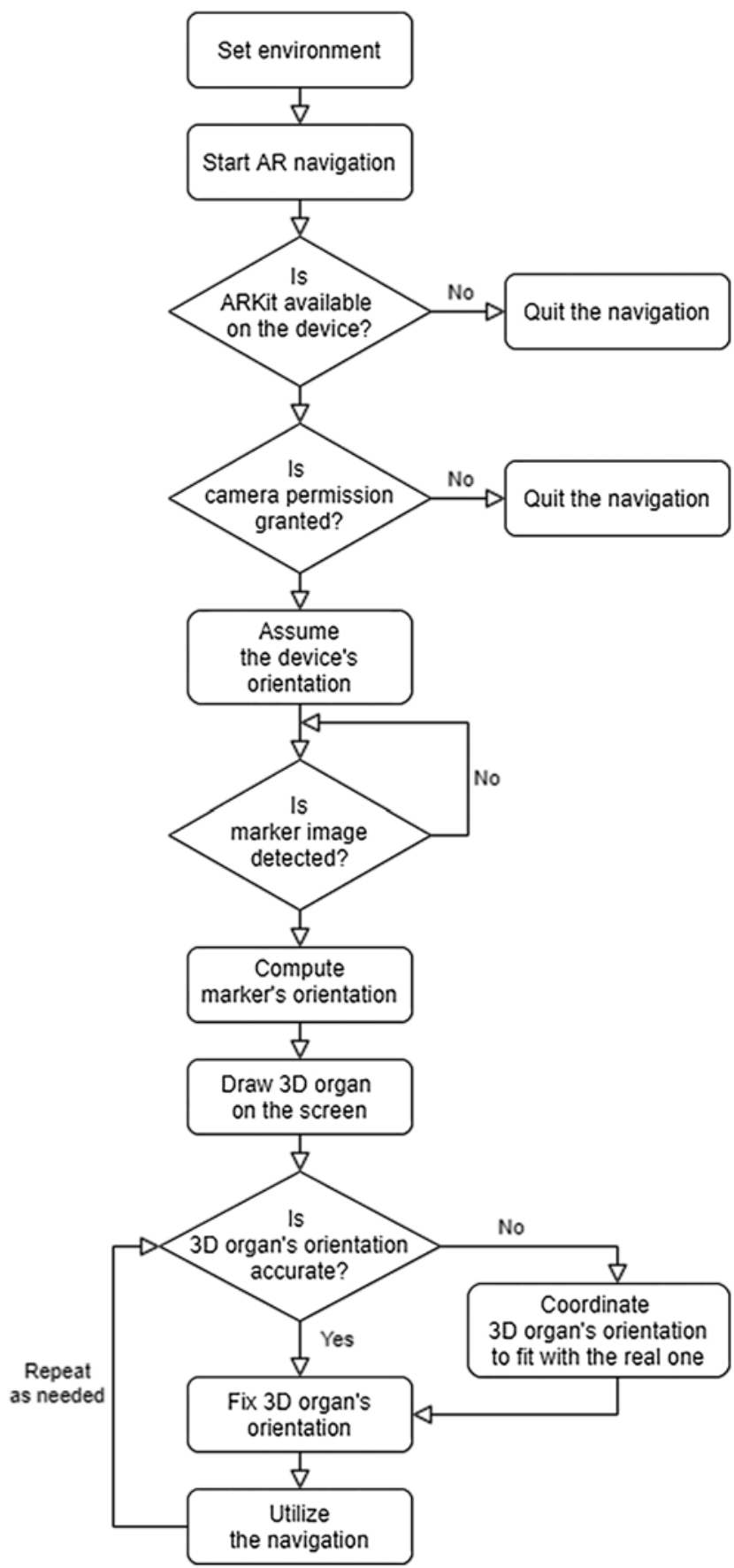

FIG. 2. Workflow of the inside-out fixed AR navigation algorithm.

environment, as described further. First, the QR (Quick Response) marker is attached to the location adjacent to the matching target that has minimal light reflection and is unlikely to be easily obscured by other objects. The QR marker holder is fabricated to ensure the placement of the marker under the same conditions when validating the registration accuracy. To minimize errors that may occur during the movement, the AR navigation visualization device (iPad) equipped with cameras and sensors is fixed in a position where the $\mathrm{QR}$ marker can be well recognized.
Running the application (software) after setting up the environment for the device and the QR marker allows the estimation of the relative position and direction of the device within the surrounding environment. This process is performed by an odometry algorithm, and there are various types of odometry algorithms depending on the data source used for calculation. The ARKit is based on visual inertial odometry (VIO), which combines visual odometry using camera images and inertial odometry using the inertial measurement unit (IMU), including an accelerometer and gyroscope (Fig. 3). VIO estimates the location of the device in the actual driving environment by setting the initial value of the device location from IMU-based data, which has a fast collection speed, and then calibrating it using camera images. Moreover, ARKit supports a loopclosure process that additionally corrects the trajectory by matching the trajectory with the starting point when moving the device and returning to the starting point during calculation with the VIO algorithm. This process is collectively referred to as visual inertial simultaneous localization and mapping (VI SLAM). The loop-closure process can be omitted due to the nature of this AR system, wherein the device operates in a fixed state.

The imaging data used for estimating the position recognized by the camera are also used for an image feature-detection algorithm that recognizes a marker, generally considering the corner and intersection of lines or the part of an image with a clear color contrast, such as black and white, as a feature point (Fig. 4) of a corresponding image to determine that the image is the same based on the location of each feature point remaining the same. The image feature-detection algorithm has high performance in texture-rich and high-contrast images, and therefore the marker image should have the same characteristics and should be made of a material that poorly reflects light and does not crumple easily to ensure undistorted image recognition by the camera. After the location of the device is determined and the QR marker is recognized, the scale of objects in the virtual space is determined by calculating the distance between the device and the QR marker from the size of the recognized marker in the preceding step. After all the requisite data are calculated, the 3D virtual model is displayed at the designated location from the marker for confirmation by the user. In case of errors in the automatically progressed registration, the user can correct the registration error by adjusting the position of the 3D virtual model using the fine-tuning function, and then fix the location of the 3D virtual model to complete the registration. The image recognition algorithm is terminated, and the application remains active without constant marker recognition.

\section{How to Run Inside-Out AR Navigation}

To create an environment similar to the surgeon's viewing angle, the visualization device (iPad) is fixed in a position that is approximately $20 \mathrm{~cm}$ away from while remaining parallel to the $\mathrm{QR}$ marker. The camera position of the device is placed in a straight line with the center point of the matching target so that the QR marker and the AR application target can be well recognized. If the matching target and the virtual 3D model were overlapped by 


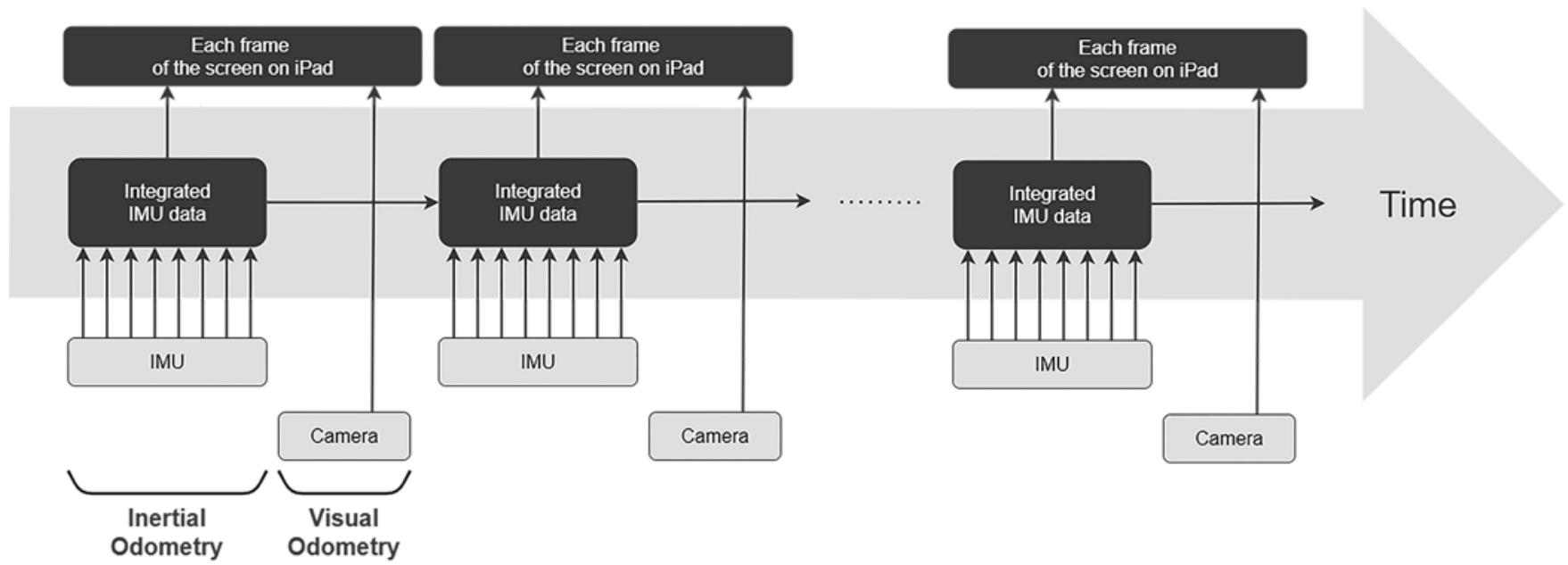

FIG. 3. Process of the VIO algorithm by camera and IMU.

the driving AR navigation, the position and angle could be corrected with the fine-tuning function, and then AR navigation could be used (Video 1).

VIDEO 1. Application of inside-out fixed AR navigation and finetuning function. The matching target (3D-printed patient model) and the virtual 3D model are overlapped by inside-out fixed AR navigation, after recognizing the QR marker and the matching target using a visualization device (iPad). The AR navigation that is distorted from the registration target is adjusted to the correct position using the fine-tuning function based on the midline (blue indicates superior sagittal sinus) and ventricle (gray) of the 3D-printed brain tumor $(r e d)$ and thalami (green) in the patient model. Copyright Chul-Kee Park. Published with permission. Click here to view.

\section{Validating the Accuracy of Inside-Out AR Navigation Registration}

The registration accuracy was validated through 2 methods: intuitive validation through visualization, and quantitative validation of matching errors by identifying

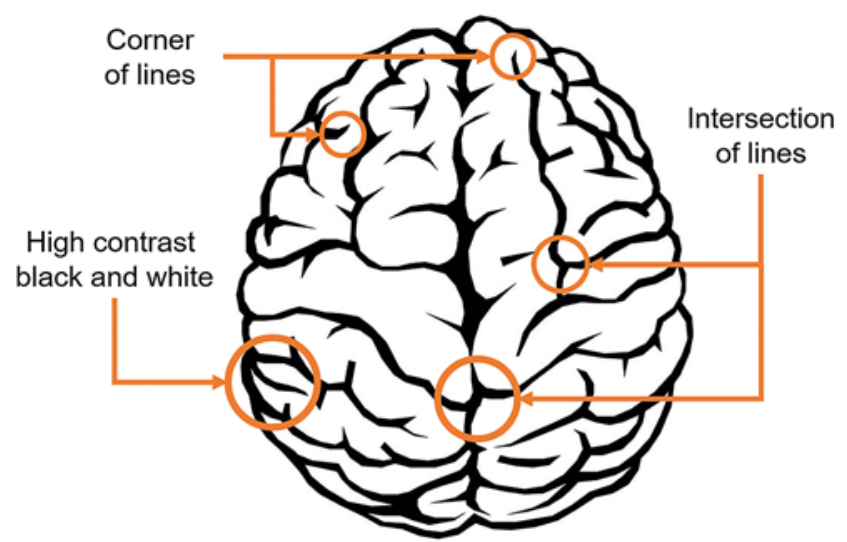

FIG. 4. QR marker of the inside-out fixed AR navigation and the feature points, such as the corner and intersection of lines or the part with clear color contrast, such as black and white colors in the image. coordinates. The patient-specific 3D-printed brain tumor and patient model were created and matched with a virtual 3D model of the same patient using AR navigation to perform visual validation. The 3D-printed model was produced in the real-life size of the actual patient and the virtual 3D model for AR navigation based on the virtual 3D model. Tumor, intracranial anatomical structures, skull, and skin were produced using 3D-printing technology (Supplemental Material). The tumor and anatomical structures were printed in different colors, the bones were white, and the skin was given the color of normal skin. To enable the identification of the intracranial space, the cranial vault was designed to open and close as required. The 3D-printed model was produced by including a fiducial marker for conventional navigation registration that was attached to the patient's skin and whose registration accuracy on the virtual 3D model of AR navigation was visually confirmed (Fig. 5A). To validate the registration accuracy of the tumor and anatomical structures, only the tumor and anatomical structures were 3D printed, and the registration accuracy was confirmed by matching the tumor and anatomical structures of the virtual 3D model of AR navigation to the tumor and the anatomical structures of the 3D-printed model after placing them exactly at the actual tumor location in the intracranial space (Fig. 5B).

The evaluation of registration accuracy using coordinates was performed using a grid and marker on which the coordinates are drawn. After placing the marker on the grid, fixing the device, and then running the AR navigation application, a translucent red square corresponding to the size and position of the marker image appeared on the screen (Fig. 5C). The accuracy was evaluated by measuring the difference between the $\mathrm{x}$ - and $\mathrm{y}$-coordinates of the square and marker image, and the difference in size of the square and marker image to determine the distance and scale error in the plane wherein the $\mathrm{x}$ - and $\mathrm{y}$-axes are visible for a total of 20 measurements. As this novel system utilizes the device in a fixed state, the evaluation of the $\mathrm{z}$-axis value (depth value) was not performed due to the 

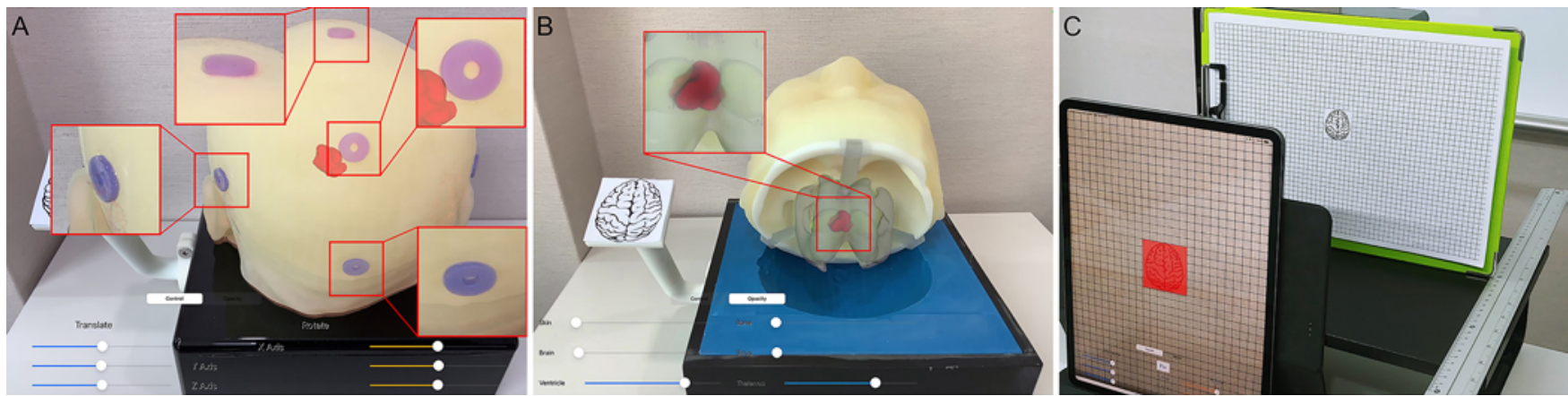

FIG. 5. A: Fiducial markers of AR navigation and the 3D-printed brain tumor and patient model are exactly overlapped. B: The tumor (red), ventricle (gray), and thalamus (green) of AR navigation are precisely overlapped with the tumor, ventricle, and thalamus of the 3D-printed brain tumor and the patient model. C: Device set at a distance of $20 \mathrm{~cm}$ from the QR marker for quantification of the registration accuracy using coordinates.

limited direction wherein the virtual 3D object could be visualized in the virtual environment.

\section{Fine-Tuning Function}

For cases when automatic registration using the QR marker is inaccurate or the registration becomes misaligned as the operation proceeds, a fine-tuning function. was developed that allows the user to adjust the position of the virtual 3D model of AR navigation. In case of a distortion in AR navigation, the user can adjust the position of the virtual 3D model of AR navigation based on anatomical landmarks (eyes, nose, and ears before surgical draping, and the superior sagittal sinus, falx, and ventricle intraoperatively). The position of the virtual 3D model can be adjusted to exactly overlap it with the matching object based on the following principles. The virtual space in AR navigation is defined such that the $\mathrm{x}-$ and $\mathrm{y}-$ axes are perpendicular to each other and create a plane and, subsequently, the 3D virtual space is constructed with the $\mathrm{z}$-axis perpendicular to the plane. The virtual space is represented on the device screen through 3 coordinate systems: local coordinate, world coordinate, and camera coordinate. The local coordinate pertains to the virtual 3D model itself and defines the orientation of the model. The world coordinate refers to the virtual space wherein the model is placed and is based on a virtual starting point at which the relative position and angle of the model can be determined. The camera coordinate refers to the coordinate system wherein the world coordinate is viewed from the standard reference of the camera. The rotation of the fine-tuning function is based on the world coordinate and the movement is based on the camera coordinate, and therefore it can always be operated in the same direction, which allows the user to intuitively determine the desired rotation and direction of movement.

\section{Opacity-Adjustment Function}

The opacity of the virtual 3D model of AR navigation that is overlapped with the registration target can be adjusted by using the opacity-adjustment function. Each of the structures, such as 3D-modeled skin, skull, brain parenchyma, intracranial anatomical structures (i.e., ven- tricle, thalamus, basal ganglia, artery, and vein), and brain tumor, can be erased or added, or the opacity can be adjusted differently while the AR navigation is in operation. These functions allow AR implementation by further emphasizing only the desired anatomical structures.

\section{Ethical Approval}

The study was conducted in accordance with the Declaration of Helsinki and complies with the current laws of the countries in which it was performed. An independent ethics committee or institutional review board (Seoul National University Hospital, IRB no. 1811-040-986, and Chungbuk National University Hospital, IRB no. 2019-06015-001) for each study site approved the study protocol.

\section{Informed Consent}

The institutional review board of Seoul National University Hospital and Chungbuk National University Hospital (Seoul National University Hospital, IRB no. 1811040-986 and Chungbuk National University Hospital, IRB no. 2019-06-015-001) waived the requirement for informed consent for the simulated clinical validation due to their being no direct interaction with patients and the retrospective nature of the study.

\section{Result}

\section{Execution of Inside-Out AR Navigation}

When AR navigation is performed through the novel application, it is overlapped and fixed onto the 3D-printed brain tumor and patient model. To complement the state of the fixed device, a setting was designed to operate through a surgeon's view and anterior, lateral, and posterior views to drive AR navigation in various directions of the registration target. The brain tumor, anatomical structures, brain parenchyma, skull, and skin that were matched from various directions can be identified separately, and the matched structures can be confirmed by erasing or adding each structure (layer) (Video 2).

VIDEO 2. Execution of inside-out fixed AR navigation. The AR navigation is driven and overlapped on the 3D-printed brain tumor and patient model. Anatomical structures can be identified by erasing 


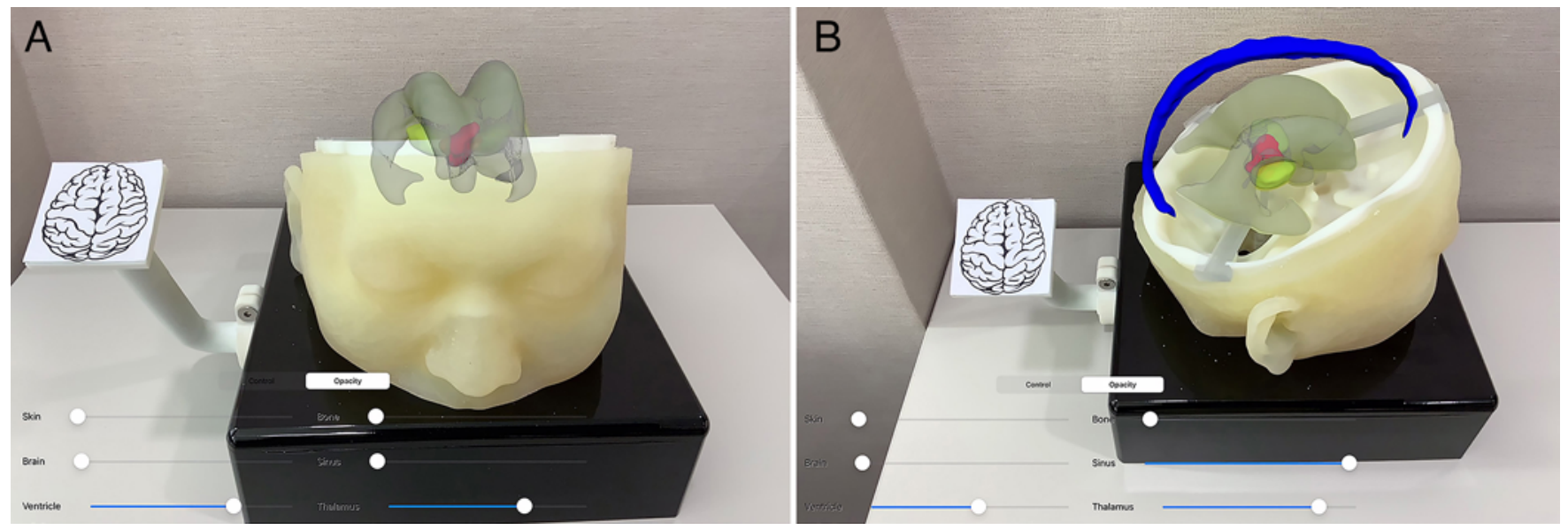

FIG. 6. Inside-out fixed AR navigation applied to the 3D-printed brain tumor and patient model shown from the anterior $(\mathbf{A})$ and lateral (B) views. Tumor (red) is located in the third ventricle (gray) between the thalami (green). The superior sagittal sinus is shown in blue.

or adding individual structures such as the superior sagittal sinus (blue), ventricle (gray), thalamus (green), and brain tumor (red).

Copyright Chul-Kee Park. Published with permission. Click here to view.

When viewed from the anterior and lateral views, AR navigation can be seen to overlap with the 3D-printed brain tumor and patient model, and the location of the tumor and the anatomical structures can be confirmed (Fig. 6A and $\mathrm{B})$.

\section{Accuracy of Inside-Out AR Navigation}

The accuracy of registration was visually identified, and the correct overlapping of the fiducial marker of AR navigation and the fiducial marker of the $3 \mathrm{D}$-printed brain tumor and patient model was confirmed at all locations without error (Fig. 5A). Furthermore, the precise overlapping of the tumor and anatomical structures of AR navigation and the tumors and structures placed in the intracranial space of the 3D-printed brain tumor and patient model were confirmed (Fig. 5B).

The device was set at a distance of $20 \mathrm{~cm}$ from the QR marker and the registration accuracy was quantified using coordinates (Fig. 5C). For the 20 measurements of movement error, the average value and standard deviation were obtained to determine the error size. The average moving errors of the $x$ - and $y$-axes were $0.52 \pm 0.35$ and $0.05 \pm$ $0.16 \mathrm{~mm}$, respectively. Moreover, the gradients from the $\mathrm{x}$ - and $\mathrm{y}$-axes were $0.35^{\circ}$ and $1.02^{\circ}$, respectively, indicating that the movement error was large in the $\mathrm{x}$-axis and the rotational error was large in the y-axis. The size of the red square generated by recognizing the markers was almost identical, which confirms that the distance between the device and the marker was relatively accurately estimated under fixed conditions.

\section{Application of the Fine-Tuning Function}

In cases of inaccurate registration after applying AR navigation, the user can apply the fine-tuning function to adjust the position of the AR navigation such that it ac- curately overlaps with the matching target. The virtual 3D model of AR navigation distorted from the registration target is adjusted to the correct position using the finetuning function based on the midline and ventricle of the 3D-printed brain tumor and patient model (Video 1).

\section{Discussion}

Patient-specific surgical planning based on individual anatomical characteristics is an important element in current and future neurosurgery. ${ }^{20-24}$ In brain tumor surgery, all patients have different tumors, in terms of size, location, extent, and characteristics, and thus personalized surgical plans are required for each patient. Despite using the most advanced neuronavigation systems for neurosurgery, neurosurgeons still require a lot of experience and training to derive an idea of the $3 \mathrm{D}$ reconstruction of lesions and structures from 2D data using their imagination. ${ }^{25}$ If AR navigation using an intuitive $3 \mathrm{D}$ model can replace neuronavigation, sophisticated personalized surgery can be accomplished more easily and even a delicate operation can be undertaken in a more generalized manner. ${ }^{14-16,26,27}$

Most of the current neuronavigation systems use an outside-in tracking method; therefore, a separate camera and sensor are required to track the spherical marker covered with retroreflective material, and a separate monitor to display a $2 \mathrm{D}$ image is required..$^{2-4}$ The introduction of a 3Dimage visualization device, such as a tablet PC and HMD equipped with a camera and sensor for tracking, has facilitated the development of a navigation system that utilizes an inside-out tracking method with relatively simple hardware. ${ }^{18,19}$ However, previous inside-out tracking AR navigation systems lacked accuracy of registration with AR images and matching targets (patients), making it difficult to apply them in actual surgeries. Therefore, we developed a QR marker-based registration method that can replace fiducial marker-based point-to-point registration and dramatically improve the registration accuracy, which is the most important issue for clinical application and shortening of registration time. Intuitive and visual validation 
confirmed by a 3D-printed patient model and quantitative validation through coordinates confirmed the registration accuracy of the novel system, and this is the feature that makes our registration technique superior to the existing registration methods. This system was developed based on ubiquitous devices, such as the iPad for inside-out tracking and visualization, and ARKit, a software manufacturing tool, for feature detection and matching of QR markers. The use of these widely available devices allows our system to operate with relatively simple equipment compared to current navigation systems. However, for scalability and versatility, there are plans to upgrade the system for universal use, with a goal to make AR navigation easy to operate in various operating systems and display devices.

The novel inside-out tracking AR navigation is operated by a method that uses the navigation system after the AR image is fixed to the matching target based on userdetermined appropriateness of the registration between the AR image and the matching target. The position of the AR image can be adjusted using the fine-tuning function not available in conventional neuronavigation before or after fixation, although the method does not correct the registration state of the AR image and the matching target in real time. Considering the possibility of intraoperative changes in the anatomical status of the patient, the utilization of AR navigation will be increased when real-time tracking is possible. In the next step, the application of VI SLAM, which is already included in the tracking and matching algorithms of this system, and the inclusion of a light detection and ranging (LiDAR) sensor for matching target recognition are ongoing to develop an AR navigation system that will further improve inside-out real-time tracking. ${ }^{17}$

To completely replace the current neuronavigation system with AR navigation, simply matching and displaying AR images to the target is not sufficient. Various auxiliary functions that the current neuronavigation system has should be installed for user convenience in the system. The opacity-adjustment function, which was developed to highlight the desired structure when making a surgical plan, is a function that to our knowledge has not been reported previously. Through this feature, the AR image can be realized by emphasizing only the desired anatomical structure, and the relative anatomical structure can be more accurately identified by easily erasing or adding the desired structure. This unique feature could be of great assistance in intuitive development of a surgical plan. When a specific location of the intracranial space of the matching target (patient) is designated with a navigation probe using the conventional neuronavigation function, the exact location, including the depth, can be confirmed on the image. This function allows identification of the extent of tumor removal during surgery and the anatomical structures of interest on demand. Moreover, a navigation-guided biopsy system for the deep lesion is an essential component that the developers intend to include. To this end, there should be an AR navigation probe that can determine the depth on an AR navigation system that functions similarly to the existing neuronavigation probe, and the depth determination must be implemented through realistic visualization. ${ }^{28}$ We are also developing an AR navigation probe that can ascertain the location, including the depth inside the AR image, by synchronizing with the inside-out tracking navigation, and a visualization method for realizing a vivid sense of depth. For clinical application after development, these functions should be validated by real-life demonstration.

We developed and installed software that includes algorithms for accurate matching based on QR markers utilizing existing hardware, which is simpler than the methods using conventional equipment, to ensure that inside-out tracking AR navigation can be directly adapted to clinical practice. As the patient-specific 3D model, including the tumor and the anatomical structure, was produced and accurately matched to the matching target, the developers expect to set up more intuitive, economical, and easy-touse neuronavigation systems in the near future to enable precise and safe personalized surgery to maximize patient benefit.

\section{Conclusions}

The inside-out tracking AR navigation system is a technology with high scalability. The successful implementation of the final product is expected to be readily applicable to neurosurgery through a variety of devices.

\section{Acknowledgments}

This work was supported by a Korea Medical Device Development Fund Grant funded by the Korean government (Ministry of Science and ICT; Ministry of Trade, Industry and Energy; Ministry of Health \& Welfare; and Ministry of Food and Drug Safety) (project number: 202012E08).

\section{References}

1. Roberts DW, Strohbehn JW, Hatch JF, Murray W, Kettenberger $\mathrm{H}$. A frameless stereotaxic integration of computerized tomographic imaging and the operating microscope. $J$ Neurosurg. 1986;65(4):545-549.

2. Kato A, Yoshimine T, Hayakawa T, Tomita Y, Ikeda T, Mitomo M, et al. A frameless, armless navigational system for computer-assisted neurosurgery. Technical note. J Neurosurg. 1991;74(5):845-849.

3. Kosugi Y, Watanabe E, Goto J, Watanabe T, Yoshimoto S, Takakura K, Ikebe J. An articulated neurosurgical navigation system using MRI and CT images. IEEE Trans Biomed Eng. 1988;35(2):147-152.

4. Maldjian JA, Schulder M, Liu WC, Mun IK, Hirschorn D, Murthy R, et al. Intraoperative functional MRI using a realtime neurosurgical navigation system. J Comput Assist Tomogr. 1997;21(6):910-912.

5. Steinmeier R, Rachinger J, Kaus M, Ganslandt O, Huk W, Fahlbusch R. Factors influencing the application accuracy of neuronavigation systems. Stereotact Funct Neurosurg. 2000; 75(4):188-202.

6. Pfisterer WK, Papadopoulos S, Drumm DA, Smith K, Preul MC. Fiducial versus nonfiducial neuronavigation registration assessment and considerations of accuracy. Neurosurgery. 2008;62(3)(suppl 1):201-208.

7. Schicho K, Figl M, Seemann R, Donat M, Pretterklieber ML, Birkfellner W, et al. Comparison of laser surface scanning and fiducial marker-based registration in frameless stereotaxy. J Neurosurg. 2007;106(4):704-709.

8. Gumprecht HK, Widenka DC, Lumenta CB. BrainLab VectorVision Neuronavigation System: technology and clinical experiences in 131 cases. Neurosurgery. 1999;44(1):97-105. 
9. Mascott CR, Sol JC, Bousquet P, Lagarrigue J, Lazorthes Y, Lauwers-Cances V. Quantification of true in vivo (application) accuracy in cranial image-guided surgery: influence of mode of patient registration. Neurosurgery. 2006;59(1)(suppl 1):ONS146-ONS156.

10. Dorward NL, Alberti O, Velani B, Gerritsen FA, Harkness WF, Kitchen ND, Thomas DG. Postimaging brain distortion: magnitude, correlates, and impact on neuronavigation. $\mathrm{J} \mathrm{Neu-}$ rosurg. 1998;88(4):656-662.

11. Liu T, Tai Y, Zhao C, Wei L, Zhang J, Pan J, Shi J. Augmented reality in neurosurgical navigation: a survey. Int J Med Robot. 2020;16(6):1-20.

12. Contreras López WO, Navarro PA, Crispin S. Intraoperative clinical application of augmented reality in neurosurgery: a systematic review. Clin Neurol Neurosurg. 2019;177:6-11.

13. Mikhail M, Mithani K, Ibrahim GM. Presurgical and intraoperative augmented reality in neuro-oncologic surgery: clinical experiences and limitations. World Neurosurg. 2019; 128:268-276.

14. Maruyama K, Watanabe E, Kin T, Saito K, Kumakiri A, Noguchi A, et al. Smart glasses for neurosurgical navigation by augmented reality. Oper Neurosurg (Hagerstown). 2018; 15(5):551-556.

15. Watanabe E, Satoh M, Konno T, Hirai M, Yamaguchi T. The Trans-Visible Navigator: a see-through neuronavigation system using augmented reality. World Neurosurg. 2016;87: 399-405.

16. Besharati Tabrizi L, Mahvash M. Augmented reality-guided neurosurgery: accuracy and intraoperative application of an image projection technique. J Neurosurg. 2015;123(1):206211.

17. Mohamed SA, Haghbayan MH, Westerlund T, Heikkonen J, Tenhunen H, Plosila J. A survey on odometry for autonomous navigation systems. IEEE Access. 2019;7:97466-97486.

18. Frantz T, Jansen B, Duerinck J, Vandemeulebroucke J. Augmenting Microsoft's HoloLens with vuforia tracking for neuronavigation. Healthc Technol Lett. 2018;5(5):221-225.

19. Hajek J, Unberath M, Fotouhi J, Bier B, Lee SC, Osgood G, et al. Closing the calibration loop: an inside-out-tracking paradigm for augmented reality in orthopedic surgery. Paper presented at: 21st International Conference on Medical Image Computing and Computer Assisted Intervention, MICCAI 2018; September 16-20, 2018; Granada, Spain.

20. Ryu WHA, Dharampal N, Mostafa AE, Sharlin E, Kopp G, Jacobs WB, et al. Systematic review of patient-specific surgical simulation: toward advancing medical education. J Surg Educ. 2017;74(6):1028-1038.

21. Sugiyama T, Clapp T, Nelson J, Eitel C, Motegi H, Nakayama $\mathrm{N}$, et al. Immersive 3-dimensional virtual reality modeling for case-specific presurgical discussions in cerebrovascular neurosurgery. Oper Neurosurg (Hagerstown). 2021;20(3): 289-299.

22. Duchin Y, Shamir RR, Patriat R, Kim J, Vitek JL, Sapiro G, Harel N. Patient-specific anatomical model for deep brain stimulation based on 7 Tesla MRI. PLoS One. 2018;13(8): e0201469.
23. van de Belt TH, Nijmeijer H, Grim D, Engelen LJLPG, Vreeken R, van Gelder MMHJ, Ter Laan M. Patient-specific actual-size three-dimensional printed models for patient education in glioma treatment: first experiences. World Neurosurg. 2018;117:e99-e105.

24. Rennert RC, Santiago-Dieppa DR, Figueroa J, Sanai N, Carter BS. Future directions of operative neuro-oncology. $J$ Neurooncol. 2016;130(2):377-382.

25. Galloway RL Jr, Maciunas RJ, Edwards CA II. Interactive image-guided neurosurgery. IEEE Trans Biomed Eng. 1992; 39(12):1226-1231.

26. Kikinis R, Gleason PL, Moriarty TM, Moore MR, Alexander E III, Stieg PE, et al. Computer-assisted interactive threedimensional planning for neurosurgical procedures. Neurosurgery. 1996;38(4):640-651.

27. Dolati P, Gokoglu A, Eichberg D, Zamani A, Golby A, AlMefty O. Multimodal navigated skull base tumor resection using image-based vascular and cranial nerve segmentation: a prospective pilot study. Surg Neurol Int. 2015;6:172.

28. Finger T, Schaumann A, Schulz M, Thomale UW. Augmented reality in intraventricular neuroendoscopy. Acta Neurochir (Wien). 2017;159(6):1033-1041.

\section{Disclosures}

Sang Joon Park is founder and CEO of MEDICALIP. Chul-Kee Park owns stock options in MEDICALIP.

\section{Author Contributions}

Conception and design: CK Park, Dho, SJ Park, Choi, Y Kim. Acquisition of data: Dho, SJ Park, Choi, Y Kim, Moon, KM Kim, Kang, Lee, MS Kim, JW Kim, YH Kim, YG Kim. Analysis and interpretation of data: Dho, SJ Park, Choi, Y Kim, Moon, KM Kim, Kang. Drafting the article: CK Park, Dho. Critically revising the article: CK Park, Dho. Reviewed submitted version of manuscript: CK Park, Dho, SJ Park. Administrative/technical/material support: SJ Park. Study supervision: CK Park.

\section{Supplemental Information \\ Videos}

Video 1. https://vimeo.com/559928124.

Video 2. https://vimeo.com/559928057.

\section{Online-Only Content}

Supplemental material is available online. Supplemental Material. https://thejns.org/doi/suppl/10.3171/ 2021.5.FOCUS21184.

\section{Correspondence}

Chul-Kee Park: Seoul National University College of Medicine, Seoul, Republic of Korea. nsckpark@snu.ac.kr. 